\title{
MODELING OF DISLOCATION DYNAMICS IN GERMANIUM CZOCHRALSKI GROWTH
}

\author{
Artemyev V. V. ${ }^{1}$, Smirnov A. D. ${ }^{1,2}$, Kalaev V. V. ${ }^{1,2}$, \\ Mamedov V. M. ${ }^{1,2}$, Sidko A. P. ${ }^{1,2}$, Podkopaev O. I. ${ }^{3}$, \\ Kravtsova E. D. ${ }^{4}$, Shimansky A. F. ${ }^{4}$ \\ 1- $\quad$ STR Group, Inc., St-Petersburg, Russia \\ 2- $\quad$ Soft-Impact, Ltd., St-Petersburg, Russia \\ 3- JSC Germanium, Krasnoyarsk, Russia \\ 4- $\quad$ Siberian Federal University, Kransoyarsk, Russia
}

Corresponding author Artemyev V.V.

Tel. +7 (812) 7031522,

Postal address: STR Group, Inc., Engels av. 27, P.O. Box 89, St-Petersburg, 194156, Russia

E-mail address: vladimir.artemyev@ str-soft.com 


\begin{abstract}
Obtaining very high-purity germanium crystals with low dislocation density is a practically difficult problem, which requires knowledge and experience in growth processes. Dislocation density is one of the most important parameters defining the quality of germanium crystal.

In this paper, we have performed experimental study of dislocation density during 4inch germanium crystal growth using the Czochralski method and comprehensive unsteady modeling of the same crystal growth processes, taking into account global heat transfer, melt flow and melt/crystal interface shape evolution. Thermal stresses in the crystal and their relaxation with generation of dislocations within the Alexander-Haasen model have been calculated simultaneously with crystallization dynamics. Comparison to experimental data showed reasonable agreement for the temperature, interface shape and dislocation density in the crystal between calculation and experiment.
\end{abstract}

\title{
Keywords:
}
A1. Dislocation density,
A1. Computer modeling,
A2. Czochralski crystal growth,
B1. Germanium 


\section{Introduction}

Semiconductor germanium crystals are used in production of wafers and substrates for electronic, infrared optics, LEDs, magnetoresistive sensors and gamma-ray detectors. The main defects of crystal structure that have significant effect on crystal quality are dislocations, which complicate using of germanium in infrared optics and eliminate possibility to manufacture substrates for $\mathrm{GaInP} / \mathrm{GaInAs} / \mathrm{Ge}$ epitaxial structures. Dislocation-free germanium is required for production of photovoltaic cells because dislocations become the reasons of mismatch between $\mathrm{Ge}$ and $\mathrm{A}^{\mathrm{III}} \mathrm{B}^{\mathrm{V}}$ compounds crystal lattices, preventing the growth of high-quality epitaxial layers [1-3]. Dislocation-free crystal growth is a technically difficult problem. In literature [1;4-7] the following general methods and approaches of obtaining of high-quality germanium are discussed: flat or slightly concave melt/crystal interface geometry during growth stage, uniformity of temperature gradient in the crystal, small radial and vertical temperature gradient in the crystal and melt etc. Experimental experience in JSC Germanium shows that mentioned criterions do not always provide quantitative characteristics of crystal quality.

The experimental trial and error approach to improve crystal growth conditions, hot zone design, and crystal temperature is expensive and takes a lot of time. Computer modeling is an alternative approach, which can be used to reduce the time and cost of experimental work for analysis and optimization of Ge crystal growth process. In the present work, we have created a computer model using special software CGSim [8] for modeling of global heat transfer, melt convection, crystal/melt interface shape evolution, thermal stress release into dislocations and performed verification of the dynamical computer model using experimental data. 


\section{Experimental approach}

The laboratory furnace REDMET for 4-inch Czochralski germanium crystal growth was used. The scheme of the furnace is presented in fig. 1. Hot zone consist of the main crucible and small additional floating crucible in the melt, side and bottom felt insulation, thermal shields and two graphite heaters. The main heater provides heating of the main crucible with germanium melt, while the additional heater, which is located above the melt free surface, provides heating of crystal periphery. The feedstock material used in the experiment was zone purified polycrystalline germanium with 6N (99.9999\%) purity. The crystal was seeded using [100] germanium seed. During Czochralski crystal growth the vertical position of the melt free surface was kept at the same level via seed holder rod and crucible pedestal moving.

The defect analysis was carried out by selective etching method using a mixture of $\mathrm{H}_{2} \mathrm{O}$, $\mathrm{HNO}_{3}$ and $\mathrm{HCl}$ in $1 \times 1 \times 2$ ratio with $0.25 \%$ of copper nitrate. The photo of the grown germanium crystal is presented in fig. 2 .

\section{Numerical model}

We used CGSim software [8] for preparing 2D axisymmetric geometry, grids and for computer modeling of heat and mass transfer, thermal stress and its release into dislocation density using the Alexander-Haasen model. Two different 2D grids in the crystal were used for investigation of grid dependence of dislocation density distribution. The initial grid consist of computational cells with dimensions $1.5 \times 2.5 \mathrm{~mm}$; the total number of cells in the crystal was 7800 . The second grid was obtained by grid refinement two times in each directions, thus the total number of cells in the crystal was increased four times and was equal to 31200 . 
We calculated crystallization dynamics of Czochralski germanium growth coupled with melt/crystal interface evolution. Governing equations are described in [8;9]. The major ways of heat transfer: conduction in the solid, melt convection and radiation were considered as follows: the View Factor approach was used for diffusive gray radiation in the furnace; the Navier-Stokes equations with the Boussinesq approximation for buoyancy effect were used for the calculation of flows. The one-equation turbulence model was used for modeling of turbulent mass and heat transfer. The wetting effect of crystal and crucible by the melt also was considered. The properties of melt/crystal used in computations presented below [10-16].

Germanium melt:

- thermal conductivity: $39 \mathrm{~W} / \mathrm{m} / \mathrm{K}$;

- density as function of temperature: $6170-0.442 \times T \mathrm{~kg} / \mathrm{m}^{3}$;

- emissivity: 0.2;

- melting temperature: $1210 \mathrm{~K}\left(937^{\circ} \mathrm{C}\right)$;

- latent heat of melting: $4.65 \times 10^{5} \mathrm{~J} / \mathrm{kg}$;

- meniscus surface tension: $0.621 \mathrm{~N} / \mathrm{m}$;

- wetting angle: $13^{\circ}$;

- heat capacity: $390 \mathrm{~J} / \mathrm{kg} / \mathrm{K}$;

- viscosity: $0.0074 \mathrm{~Pa} \cdot \mathrm{s}$;

- temperature coefficient of surface tension: $7.32 \times 10^{-5} \mathrm{~N} / \mathrm{m} / \mathrm{K}$.

Germanium crystal:

- conductivity: $17 \mathrm{~W} / \mathrm{m} / \mathrm{K}$;

- emissivity: 0.55;

- density as function of temperature: $5351-0.094 \times T \mathrm{~kg} / \mathrm{m}^{3}$;

- heat capacity: $390 \mathrm{~J} / \mathrm{kg} / \mathrm{K}$. 
The thermal stress and dislocation density were calculated in the crystal using Alexander-Haasen model [17]. During crystal growth, the thermal stress is increasing due to temperature variation in the crystal and high stress results in the inelastic creep deformation. The total strain is assumed to consist of components:

$$
\varepsilon=\varepsilon^{\mathrm{e}}+\varepsilon^{\mathrm{T}}+\varepsilon^{\mathrm{c}}
$$

here $\varepsilon^{\mathrm{e}}, \varepsilon^{\mathrm{T}} \varepsilon^{\mathrm{c}}$ are elastic, thermal, creep strain respectively.

Thermal strain is produced by thermal expansion:

$$
\varepsilon^{\mathrm{T}}=\beta \times\left(\mathrm{T}-\mathrm{T}_{\mathrm{ref}}\right)
$$

here $\beta$ is the volumetric thermal expansion coefficient, $\mathrm{T}$ is the local temperature, $\mathrm{T}_{\text {ref }}$ is the reference temperature.

The Hook's law shows the dependence between the elastic strain and stress in the crystal:

$$
\sigma_{\mathrm{ij}}=\sum_{\mathrm{j}} \mathrm{c}_{\mathrm{ij}} \times \varepsilon_{\mathrm{ij}}^{\mathrm{e}}
$$

here $\mathrm{c}_{\mathrm{ij}}$ is the stiffness matrix.

The creep strain rate is proportional to the local dislocation density $\mathrm{N}_{\mathrm{m}}$, averaged velocity for all moving dislocations $\mathrm{v}$ and Burgers vector $\mathrm{b}$ [17-19]:

$$
\dot{\varepsilon}^{\mathrm{c}}=\mathrm{b} \times \mathrm{v} \times \mathrm{N}_{\mathrm{m}}(4)
$$

The velocity $\mathrm{V}$ is proportional to the effective stress $\tau_{\text {eff: }}$

$$
\begin{gathered}
\tau_{\mathrm{eff}}=\sqrt{\mathrm{J}_{2}}-\mathrm{R} \times \frac{\mathrm{E} \times \mathrm{b}}{4 \times \pi \times\left(1-v^{2}\right)} \times \sqrt{\mathrm{N}_{\mathrm{m}}} \\
\mathrm{v}=\mathrm{k}_{0} \times \tau_{\mathrm{eff}}^{\mathrm{p}} \times \exp \left(-\frac{\mathrm{Q}}{\mathrm{k} \times \mathrm{T}}\right)
\end{gathered}
$$


here $J_{2}$ is the second invariant of the tensor $S_{i j}=\sigma_{i j}-1 / 3 \times \delta_{i j} \times \sum_{k} \sigma_{k k}$; $R$ is the relative hardening factor; E is the Young's modulus; $v$ is the Poisson's ratio.

The effective stress is equal to the difference of applied stress and internal stress provided by the strain hardening effect: increased dislocation density creates a barrier for dislocation movement. In case $\tau_{\text {eff }}<0$ dislocations are immobilized and hence do not multiply.

The mobile dislocation density multiplication rate is proportional to mobile dislocation density as follows:

$$
\dot{\mathrm{N}}_{\mathrm{m}}=\mathrm{K} \times \mathrm{k}_{0} \times \tau_{\mathrm{eff}}^{\mathrm{p}+\mathrm{l}} \times \exp \left(-\frac{\mathrm{Q}}{\mathrm{k} \times \mathrm{T}}\right) \times \mathrm{N}_{\mathrm{m}}
$$

here $\mathrm{K}, \mathrm{k}_{0}, \mathrm{p}, \mathrm{l}$ are material constants, $\mathrm{Q}$ is the Peiersl potential, $\mathrm{k}$ is the Boltzman constant, $\delta_{\mathrm{ij}}$ is the Kronecker delta.

In the table 1 the parameters of the Alexander-Haasen model for germanium are given, which were calculated from data in [18-20].

Table 1. Parameters of Alexander-Haasen model

\begin{tabular}{|c|c|}
\hline Burgers vector $\mathrm{b}, \mathrm{m}$ & $3.98 \times 10^{-10}$ \\
\hline Relative strain hardening factor $\mathrm{R}$ & 0.723 \\
\hline Material constant $\mathrm{p}$ & 1.7 \\
\hline Material constant $\mathrm{l}$ & 1 \\
\hline Material constant $\mathrm{k}_{0}, \mathrm{~m}^{4.4} / \mathrm{N}^{1.7} / \mathrm{s}$ & $6.76 \times 10^{-8}$ \\
\hline Material constant $\mathrm{K}, \mathrm{m} / \mathrm{N}$ & $1.07 \times 10^{-3}$ \\
\hline Peierls potential $\mathrm{Q}, \mathrm{eV}$ & 1.62 \\
\hline Young's modulus $\mathrm{E}, \mathrm{Pa}$ & $10.3 \times 10^{10}$ \\
\hline Poisson's ratio $\mathrm{v}$ & 0.26 \\
\hline
\end{tabular}


The initial dislocation density in the Ge seed is equal $\mathrm{N}_{0}=11 / \mathrm{cm}^{2}$.

\section{The results and discussion}

Crystal growth and crystal cooling stages with PID-algorithm of heater power adjustment were simulated by software CGSim, providing information about global temperature distribution, thermal stress in the crystal, melt convection and melt/crystal interface shape evolution.

First, let us consider predictions of temperature distribution. Crystal quality depends on the temperature distribution, which is determined by a hot zone design, heater power, crystal pulling rate and cooling stage. Verification of the computer model showed the temperature difference in the thermocouple less than $10 \mathrm{~K}$ between the experiment data and calculation results. In fig. 3 (left) the thermocouple temperature distribution near the additional heater is illustrated for experiment and simulation. The deflection of the melt/crystal interface is predicted within $1 \mathrm{~mm}$ accuracy. During pulling process, the interface shape is changed from concave for upper crown to $\mathrm{V}$-shape for body stage. The experimental interface deflection in the center is $2.5 \mathrm{~mm}$ compared to calculated interface deflection in the center of about 2.3 $\mathrm{mm}$, details of interface geometry are presented in fig. 3 (right).

Second, using the Alexander-Haasen model, we obtained the data for dislocation density and stress distribution in the crystal. Analyzing the equations of $\mathrm{AH}$ model, we have found two factors, which determine dislocation multiplication during growth and cooling stages: absolute temperature and thermal stress in the crystal. In fig. 4 distributions of the thermal stress at the left side and dislocation density rate at the right side for crown growth stage are illustrated. The maxima of the thermal stress correspond to maxima of the dislocation density multiplication rate, so the reduction of the thermal stress is important to 
obtain low-dislocation germanium crystal. In the upper crystal part, the dislocation density multiplication rate is about zero due to low stress and lower crystal temperature.

Cooling stage after growth is characterized by relatively low crystal temperature with other thermal stress distribution and maxima of dislocation density multiplication rate than for growth stage. We observed that the dislocation density is increased by $28 \%$ in the crystal center and by $38 \%$ at the crystal periphery during crystal cooling. Comparison of the dislocation density distribution between initial and refined grids shows $10 \%$ difference.

Experimental data on dislocation distribution in the germanium crystal were obtained in several points in two horizontal cross-section in the middle and lower parts of the crystal body at different radial positions. Typical W-shape radial distribution of the dislocation density is observed in the horizontal cross-section: from $11001 / \mathrm{cm}^{2}$ at the center with decrease to 600 $1 / \mathrm{cm}^{2}$ at half of the crystal radius, and then increase to $10001 / \mathrm{cm}^{2}$ at the crystal periphery. During growth and cooling stages, the maxima of thermal stress were observed at the crystal center and periphery of the crystal, so the high multiplication rate of dislocations was concentrated in these regions, which explains dislocation density $\mathrm{W}$-shape across crystal.

In the work [21] we presented so-called simple method of dislocation transfer at the growth interface during Czochralski growth simulation. The idea of this method is that dislocations are moving along vertical grid lines at the interface during crystal pulling. In fig. 5, a part of crystal grid with the melt/crystal interface shape and two arrows are presented. The blue arrow 1 in fig. 5 corresponds to the direction of dislocation evolution in a local point of the interface using the simple method. The disadvantage of such algorithm is that the dislocation density depends on numerical parameter, grid structure in the crystal. To improve it we present a more advanced physical algorithm in the current work, which is characterized by dislocation transfer to the newly grown layer perpendicular to the interface geometry in each point. Such approach is illustrated by red arrow 2 in fig. 5 for a particular point of the 
interface. In fig. 6, the dislocation density distribution in the crystal calculated using both approaches are given. The radial dislocation density distribution obtained from experiment and calculation results are presented in fig. 7, the deviation of calculations is about $30 \%$. We demonstrated that advanced method of dislocation evolution has better agreement with experimental data than the simple method.

\section{Conclusion}

We have performed comprehensive computer simulation of 4-inch Czochralski germanium crystal growth using CGSim software. Verification of the computer model shows good agreement between calculation and experimental data for temperature measurements by thermocouple and interface shape deflection.

For investigation of grid dependence initial crystal grid with 7800 cells and refined grid with 31200 cells were used in the crystal domain. The difference of crystal temperature, melt/crystal interface deflection and dislocation density between initial and refined grids is less or about $10 \%$, so using the initial grid is enough to obtain reasonable predictions.

The thermal stress and crystal temperature are main factors, affecting dislocation density distribution in the crystal. The absolute value of the dislocation density is determined not only by growth stage but also by crystal cooling stage, when dislocation density increases by about $30 \%$.

We presented a new modified method of dislocation density transfer to a newly grown layer during growth simulation, which is founded on dislocation movement along the vector normal to the melt/crystal interface in each point. Comparison with experiment showed a reasonable agreement both for absolute values of dislocation density in the crystals, and radial distributions of the dislocation density in the middle and lower parts of crystal body with $30 \%$ 
accuracy. The results have proved the suitability of the Alexander-Haasen model for prediction of the dislocation density in germanium crystals. 


\section{References}

1. Claeys Cor L. Germanium-based technologies: from materials to devices. Berlin [etc.]: Elsevier, 2007. 449 p.

2. Depuydt B., Theuwis A., Romandic I. Materials Science in Semiconductor Processing. 2006. V.9. Issues 4-5, P. 437-443.

3. Dimroth F., Kurtz S. MRS Bull. 2007. 32 (3). P. 230-235.

4. Wang G., Sun Y., Xiang W., et al. Journal of Crystal Growth. 2012. 32. P. 27-30.

5. Konakov P. K., Verevochkin G.E. et al. Crystal growth heat and mass transfer, Moscow, Metallurgy, 1971 (in Russian)

6. Moskovskih V. A., Kasimkin P. V., Shlegel V. N., Vasiliev Y. V., Gridchin V. A., Podkopaev O. I. Journal of Crystal Growth. 2014. 401. P. 767-771.

7. Podkopaev O. I., Shimansky A.F., Low impurity and dislocation density germanium crystal growth, Monography, Krasnoyarsk, 2013 (in Russian)

8. CGSim software http://www.str-soft.com/products/CGSim/

9. Kalaev V.V., Evstratov I. Yu., Makarov Yu. N. Journal of Crystal Growth. 2003. 249. P. 87-99

10. Rupp R. Muller G. Journal of Crystal growth. 1991. 113. P.131-139

11. Artemyev V.K., Folomeev V.P., Ginkin V.P., Kartavykh A.V., Milvidskii M.G., Rakov V.V. Journal of Crystal Growth. 2001. 223. P. 29-37

12. Sampath R., Zabaras N. Journal of Physical Chemistry B. 2001. 105. P. 121126-12133

13. Patzold O., Fischer B., Croll A. Crystak Research and Technology. 2002. 37. P. 10581065

14. Van den Bogaert N., Dupret F. Journal of Crystal Growth. 1997. 171 P. 65-76

15. Nakanishi H. Journal of Crystal Growth. 1998. 191. P. 711-717

16. Kuandakov L.L. Journal of Crystal Growth. 2001. 222 P. 852-861 
17. Alexander H., Haasen P. Solid State Phys. 1969. 22 P. 27-158

18. Sumino K., Kodaka S. Materials Science and Engineering. 1974. 13 P. 263-268

19. Sumino K. Materials Science and Engineering. 1974. 13. P. 269-275

20. Ioffe Institute site http://www.ioffe.ru/SVA/NSM/Semicond/Ge/mechanic.html

21. Podkopaev O.I. Artemyev V. V., Smirnov A. D., Kalaev V. V. ,Mamedov V. M., Sidko A. P. Kravtsova E. D., Shimansky A. F., Technical Physics. 2016, vol. 86, issue 9 
Figures

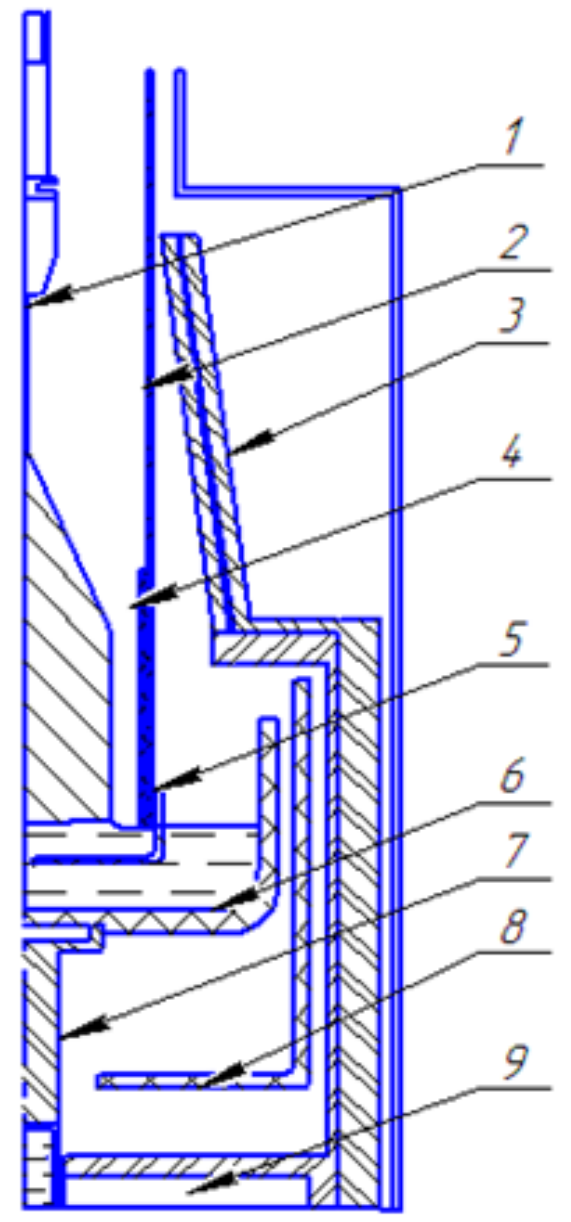

Fig. 1. 


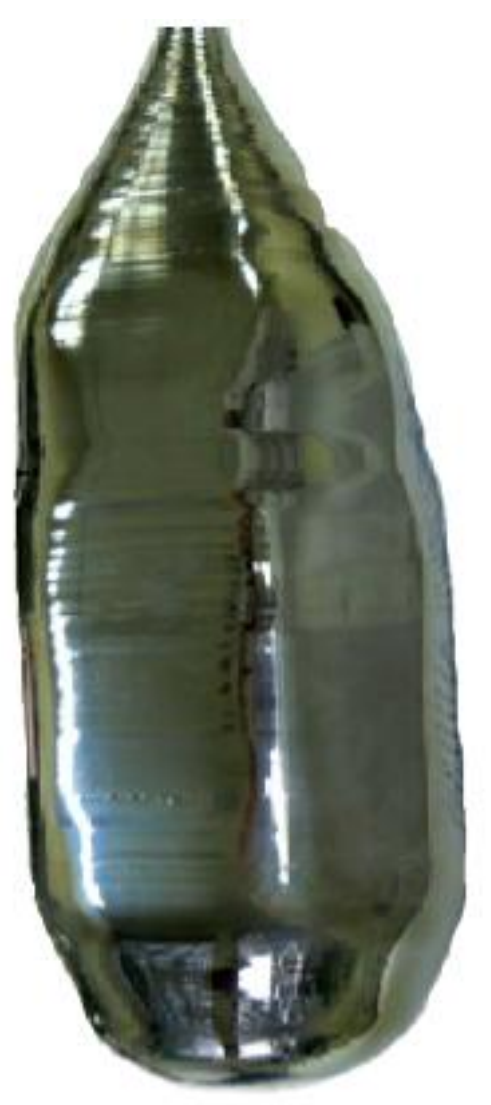

Fig. 2
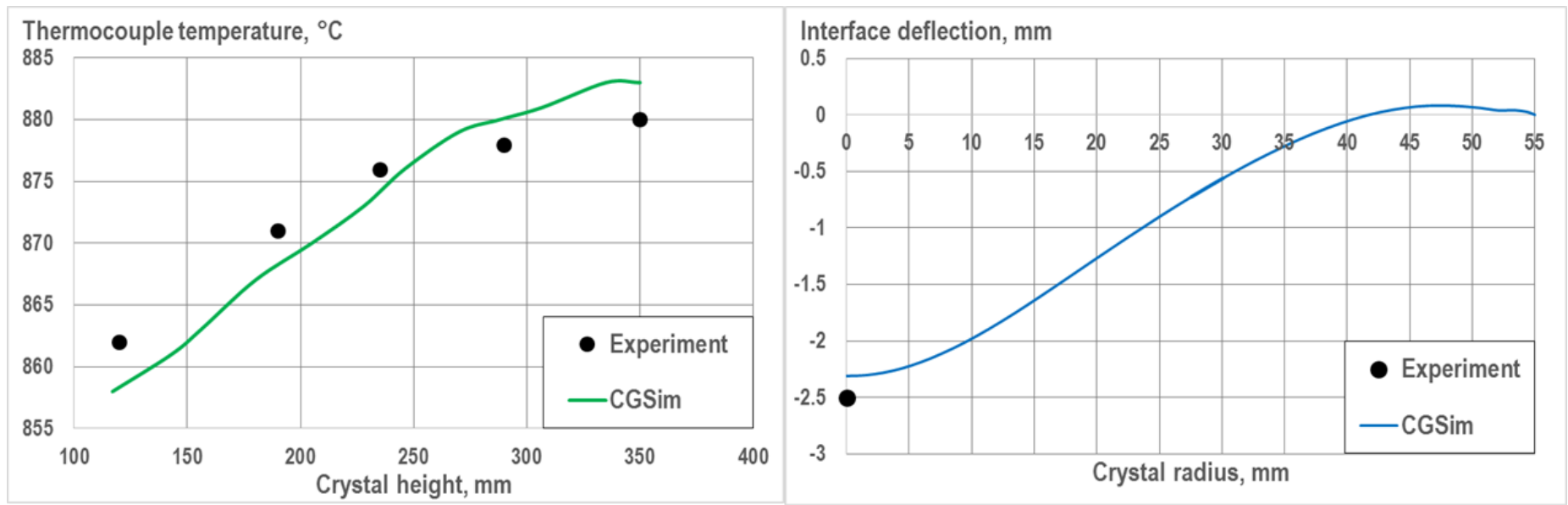

Fig. 3 


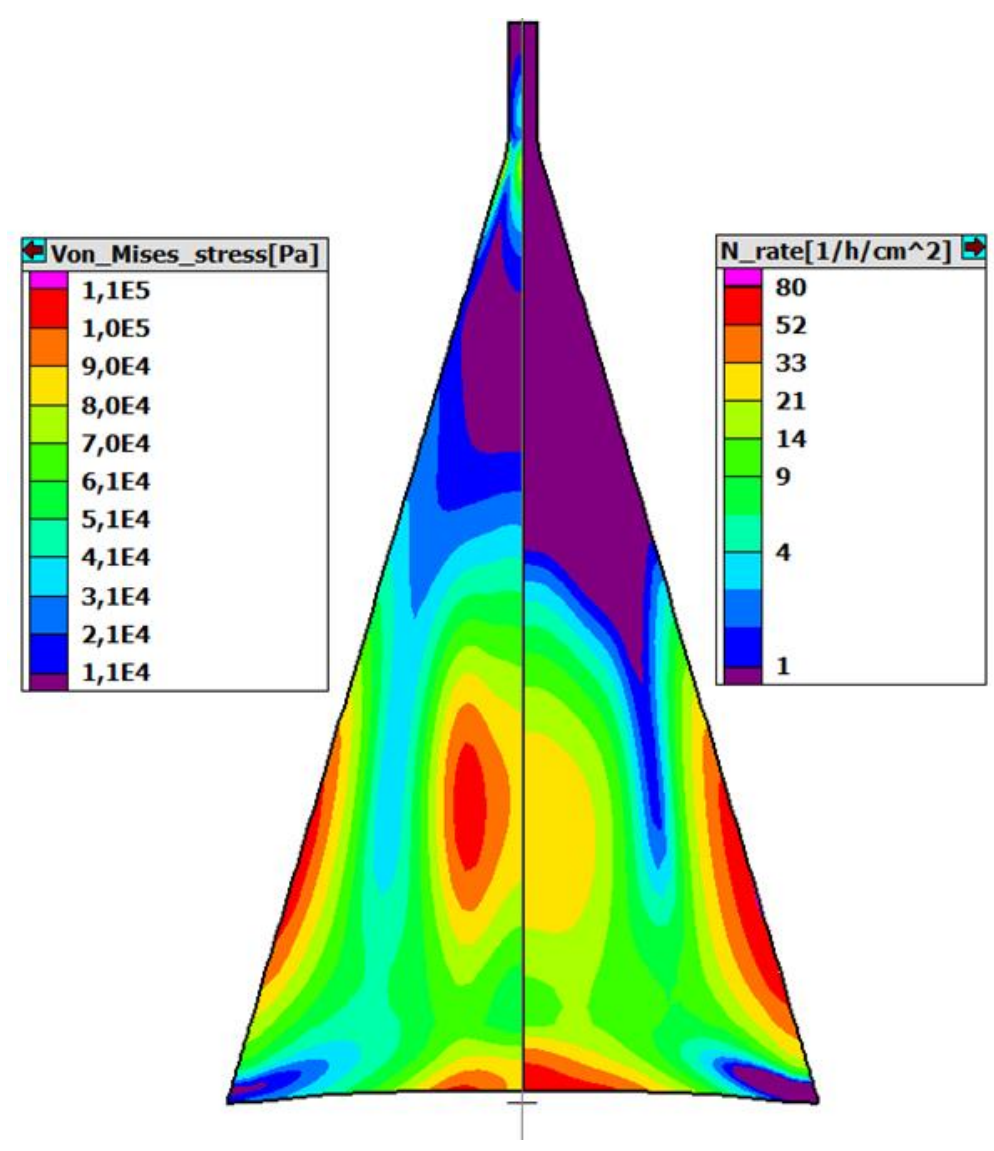

Fig. 4

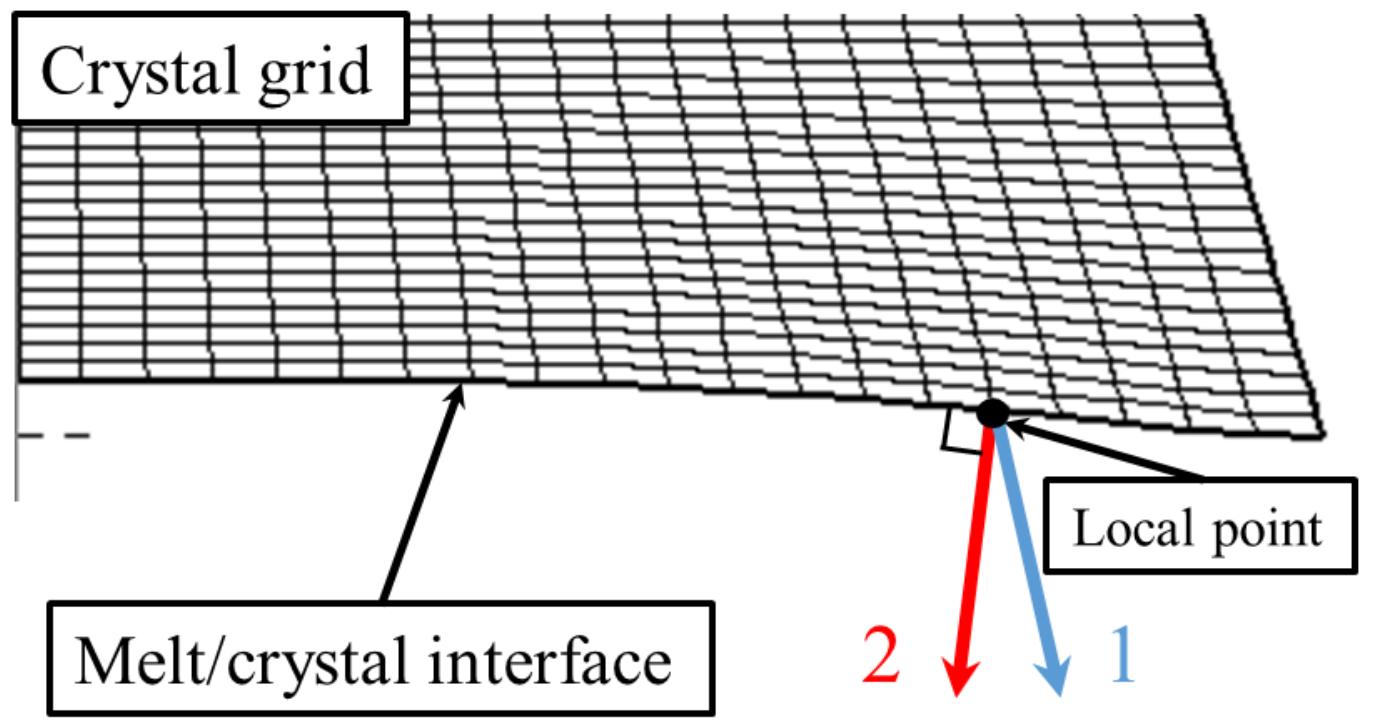

Fig. 5 


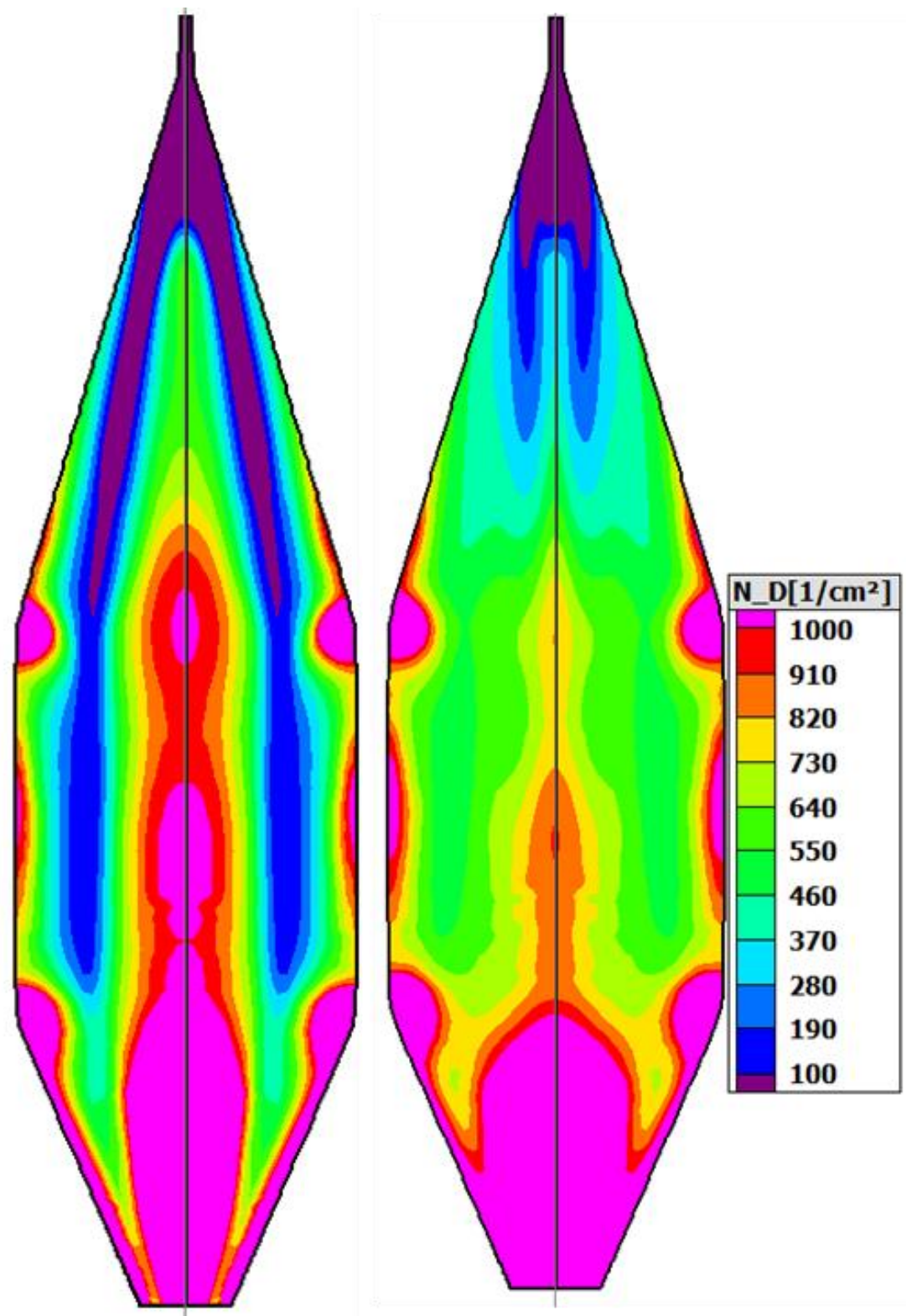

Fig. 6 

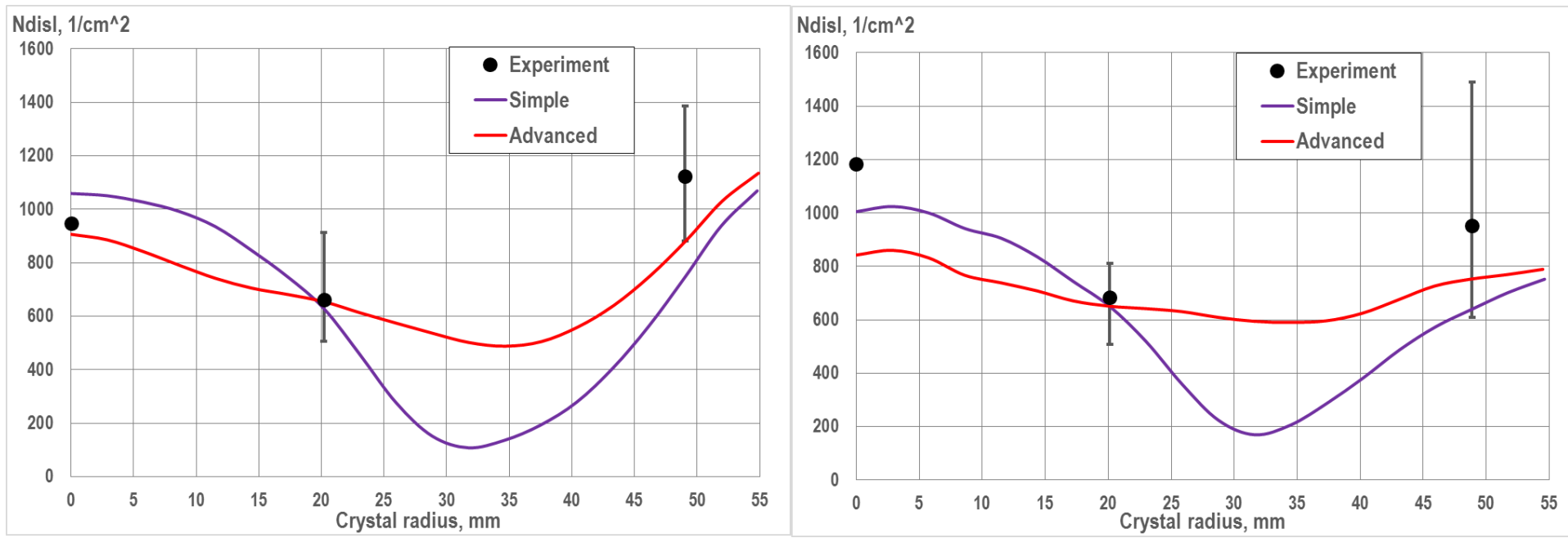

Fig. 7 


\section{Figure captions}

Fig. 1. The scheme of growth furnace:

1 - holder; 2 - quartz shield; 3 -cone shield;

4 - additional heater; 5 - inner crucible; 6 - main crucible;

7 - pedestal; 8 - main heater; 9 - graphite felt

Fig. 2. 4-inch germanium crystal

Fig. 3 Thermocouple temperature (left) and melt/crystal interface deflection (right)

Fig. 4. Thermal stress (left) and dislocation density multiplication rate (right)

Fig 5. The schemes of dislocation evolution at the melt/crystal interface

Fig. 6. Dislocation density distribution using simple method (left) and advanced method (right)

Fig. 7. Radial dislocation density distribution at the middle part (left) and lower part (right) of crystal body 